\title{
EXPLORANDO EN LA IDENTIDAD: ¿COMO ENFRENTAN LOS DOCENTES UNIVERSITARIOS LOS INCIDENTES CRITICOS QUE OCURREN EN LAS AULAS DE FORMACION DE FUTUROS PROFESORES?
}

\author{
EXPLORING IN THE IDENTITY: HOW DO UNIVERSITY PROFESSORS \\ FACE THE CRITICAL INCIDENTS THAT OCCUR IN THE CLASSROOMS DURING \\ THE EDUCATION OF FUTURE TEACHERS?
}
Explorando a identidade: Como professores universitários enfrentam os incidentes críticos que ocorrem nas aulas de formação de futuros professores?

\author{
Claudia Contreras Contreras ${ }^{1}$, Carles Monereo Font ${ }^{2}$, Antoni Badia Garganté ${ }^{3}$ \\ 1claudiacontreras.contreras@gmail.com, \\ ${ }^{2}$ Universitat Autònoma de Barcelona. carles.monereo@uab.cat, \\ ${ }^{3}$ Universitat Oberta de Catalunya. tbadia@uoc.edu
}

\begin{abstract}
RESUMEN
A partir de las entrevistas realizadas a cinco docentes universitarios chilenos que forman a futuros profesores, el presente estudio se propone responder principalmente a tres preguntas: ¿cómo definen su identidad los profesores universitarios que forman a futuros maestros?, ¿cuáles son los incidentes críticos comunes en sus clases? y ¿cómo los enfrentan? En relación a estos cuestionamientos, se elabora un análisis que permite identificar elementos relevantes en su identidad, así como algunas variables asociadas a los incidentes críticos descritos. Los resultados permiten presentar un conjunto de categorías desde las cuales se evidencian distinciones en la identidad docente de este grupo de profesorado, comunicar evidencias sobre las formas de actuación de los docentes ante incidentes críticos y visualizar un potencial de uso importante de estos eventos, en calidad de instrumento para la formación permanente. Finalmente se plantean algunos alcances y limitaciones de la presente investigación en vista a próximos estudios.
\end{abstract}

Palabras clave: Formación permanente del profesorado universitario, identidad docente, incidente crítico, formación inicial docente.

\begin{abstract}
This study intends to respond mainly to three questions relying on the interviews held with five Chilean university professors that train future teachers. These questions are: 'How do university professors who train future teachers define their identity?', 'What are the common critical incidents in their classes?', and, 'How do they deal with these critical incidents?' An analysis based on these questions is made that identifies relevant elements in their identity, as well as some variables associated to critical incidents they described. The results allow a set of categories from which identity differences of the professors of this faculty group can be drawn. In the same way, they show evidence of the ways professors perform facing critical incidents, and visualize an important potential use of these events as an instrument for the continuous education. Finally, some scopes and limits of this research are outlined which need further investigation.
\end{abstract}

Key words: continuous education of university professors, teacher identity, critical incident, initial teacher education.

\section{RESUMO}

A partir de entrevistas realizadas com cinco professores de universidades chilenas que formam a futuros professores, este estudo pretende responder a três questões principais: 'Como professores universitários, que formam a futuros docentes, definem sua identidade?', 'Quais são os incidentes críticos comuns em suas aulas?' e 'Como 
eles enfrentam esses incidentes críticos?'. Em relação a esses questionamentos, se elabora uma análise para identificar os elementos relevantes em sua identidade, assim como algumas variáveis associadas aos incidentes críticos descritos. Os resultados permitem apresentar um conjunto de categorias, desde as quais se evidenciam distinções na identidade docente deste grupo de professores, comunicar evidencias nas formas de atuação dos docentes frente a incidentes críticos e mostrar um uso potencial importante destes eventos, como uma ferramenta para a aprendizagem ao longo da vida. Finalmente, são expostas algumas possibilidades e limitações da presente investigação em vista aos estudos futuros.

Palavras-chave: formação permanente de professores de universidade, identidade docente, incidente crítico, formação inicial de profesores.

\section{MARCO TEORICO}

A continuación se presenta la discusión bibliográfica que constituye el marco conceptual del presente estudio. Este marco aborda de manera breve la formación permanente del profesorado universitario como un tema de preocupación actual, y la identidad profesional y los incidentes críticos como vías para la formación docente.

\subsection{EL PROFESORADO Y LA FORMACION PERMANENTE EN EL CONTEXTO UNIVERSITARIO}

Hoy en día los cambios políticos, económicos y sociales globalizados han derivado en múltiples transformaciones, como la inmediatez en el acceso a la información, el aumento en la cobertura de la educación superior y en la multiplicación de roles del profesorado, entre otros aspectos. A partir de estos cambios se comprende que haya un impacto en las necesidades y funciones de la educación en todos sus niveles, y por tanto también en la universidad, teniendo implicaciones en los modelos de formación que se ofrecen a los universitarios y profesores, influyendo en el currículo, en las prácticas de aula y en el ejercicio docente.

Desde esta perspectiva, cabe preguntarse si actualmente se desarrollan propuestas de formación permanente que permitan al profesorado universitario responder a las principales contingencias existentes en la educación superior. Más aún cuando se piensa que un número importante de profesionales que ejercen la docencia en la universidad no han hecho una especialización pedagógica. A esto se suman algunos antecedentes que indican que al contrastar las concepciones de enseñanza de docentes universitarios, con las de maestros y profesores de otros niveles educativos, éstas difieren, ya que los primeros no suelen asumirse como enseñantes, sino más bien como profesionales expertos en un ámbito disciplinar específico (De la Cruz, Pozo, Huarte, Scheuer, 2006).

En continuación a lo anterior, y teniendo como foco el desempeño del profesor universitario, se dice que existe consenso en señalar que la enseñanza es de calidad cuando los docentes despliegan los conocimientos necesarios para enfrentar las tareas y resolver los problemas propios del contexto donde enseñan (Avalos, 2002). A su vez se espera que este desempeño impacte positivamente en los aprendizajes del estudiante, lo cual difícilmente se logra si no se cuenta con las competencias y estrategias de enseñanza adecuadas. En complemento, algunos autores establecen que un buen profesor es aquel que es capaz de construirse a través de múltiples versiones de sí mismo, consiguiendo con esto flexibilizar y ajustar en diferentes contextos educativos, las concepciones, estrategias y sentimientos que constituyen sus discursos y actuaciones como enseñante (Monereo y Badia, en prensa).

Por otro lado, en el marco del Espacio Europeo de Educación Superior (EEES), se espera que, en su rol de profesor, el docente universitario posea las competencias para 
desarrollar metodologías activas de enseñanza e incluya una evaluación formativa clara y coherente con los objetivos de aprendizaje. En particular, dentro de esta expectativa se considera además que antes de realizar su asignatura, el profesor planifique las experiencias de enseñanza y aprendizaje, las que luego deberá motivar, evaluar y retroalimentar, entre otros aspectos esperables de su desempeño (Benito y Cruz, 2005). Considerando los requisitos aquí planteados, y que la docencia es una tarea compleja y contextualizada, cabe preguntarse nuevamente si la formación permanente en la docencia universitaria ofrece las posibilidades para responder a los desafíos detallados en el presente texto, más aún cuando dentro de la sala de clases (y a veces fuera de ella) el profesional está ejerciendo un rol como profesor que lo responsabiliza de las situaciones y relaciones construidas para que otros aprendan.

En el ámbito universitario resulta habitual que los profesores se desempeñen en distintos roles los cuales pueden ser complementarios o conducirse por líneas paralelas. De esta manera, se comprende que el profesor se movilice en un continuo de tareas asignadas, como profesional experto, docente, investigador y/o gestor. En la interacción de estos roles resulta esperable que algunos tengan mayor importancia y espacio, dependiendo de la institución, los intereses y experiencias del profesor; sin embargo, es sabido que tradicionalmente las instituciones de educación superior han privilegiado el papel de investigador por sobre otros. Siguiendo esta línea, resulta una ambición legítima pensar que los cuatro roles antes mencionados puedan armonizarse, de manera tal que la enseñanza como elemento central del trabajo docente resulte enriquecida y desafiada a partir de las contribuciones que puedan derivarse de las otras funciones que el profesor desarrolla en su institución educativa (Perales, Sánchez y Chiva, 2002).

A lo largo de las últimas décadas se ha establecido que una buena enseñanza necesita aproximaciones auténticas, metodologías activas y una evaluación formativa que demanda del profesor la utilización de conocimientos y habilidades específicas para resolver en terreno las tareas que se le presentan (Coll, Palacios, Marchesi, 2008). Siendo así, puede afirmarse que la formación permanente del profesorado universitario debe ser diseñada e implementada en condiciones que aumenten las posibilidades de que los docentes desarrollen las competencias para construir conocimiento, afrontar incidentes y mejorar su enseñanza.

Una visión que parece ajustarse a estos requerimientos en la formación permanente es lo que plantea el enfoque de la práctica reflexiva (Navarro, López y Barroso, 1998; Perrenoud, 2007). Este enfoque sugiere que a través de un adecuado trabajo personal el docente accede a un conocimiento profesional distintivo, que puede desarrollarse en cualquier contexto, pero que a la vez exige un esfuerzo para explicitarlo y sistematizarlo, de manera que la experiencia pueda ser integrada para su uso estratégico. Este enfoque puntualiza que la reflexión personal, a partir de la experiencia, puede ser comunicada y compartida en una comunidad, pudiendo también usarse otros procedimientos más individuales que servirán a propósitos como la explicitación y la toma de conciencia de los procesos involucrados en el desempeño docente (Martin y Cervi, 2006).

Desde una mirada más bien crítica puede señalarse que una formación genérica no será suficiente para alcanzar una explicitación y un camino para la transformación, puesto que muchas veces no ofrecerá un andamiaje, un contexto, ni supondrá una evolución en las actuaciones y concepciones. Si bien es cierto la reflexión podría ser un primer paso en un trabajo de formación docente, su uso no asegurará un proceso de transformación 
en el conocimiento, en las representaciones y las prácticas. Para esto se cree necesario un trabajo personal situado, un modelo referencial y una revisión de las propias teorías implícitas acerca de enfoques, roles y otros constructos que influyen en las respuestas o actuaciones del profesor en el aula. Esto supondrá la apertura tanto hacia el uso de las habilidades cognitivas como sociales, comunicativas y emocionales de los docentes, sin dejar de lado que este profesional debe ser además un experto en contenidos, procesos, metodologías, en sus alumnos y sí mismo.

Incentivar el desarrollo del conocimiento profesional y de las habilidades requeridas en la docencia implica dejar atrás una concepción rígida, homogénea y descontextualizada de la formación, orientándola hacia un enfoque diversificado y auténtico, que repercuta en la práctica docente y en las posibilidades de éxito de los enseñantes y sus aprendices.

A partir de lo anterior, resulta importante poder revisar los referentes que han guiado hasta hoy las propuestas en formación del profesorado, no para descartarlos, sino más bien para integrarlos o releerlos desde una perspectiva de la formación permanente del profesorado universitario que se sitúe lejos de aislamiento y la introspección extrema (Day, 2006).

Si tenemos claro que la docencia en general -y la universitaria con mayor razóntiene que ir más allá de la clase magistral deben abrirse nuevas alternativas para que el profesor tenga un rol protagónico en su propia formación, puesto que tal como sus alumnos al enfrentarse al reto de aprender será él quien deberá ocupar un rol activo en la revisión y construcción de sus aprendizajes.

Una propuesta que permite situar y reafirmar las condiciones adecuadas para la formación permanente del profesorado universitario en la actualidad es la que ofrece el enfoque constructivo o estratégico de la formación docente. Este enfoque, que se define a partir de ciertos criterios y características psicológicas y sociocognitivas, establece que los conocimientos implicados en la formación deben emerger de la propia experiencia y práctica educativa, para cuyo análisis resulta fundamental el desarrollo y uso de habilidades metacognitivas, así como la mediación dialógica del formador, con la teoría, la praxis y los sujetos en formación. A partir de ello, esta perspectiva entiende que para la formación del profesorado deben ponerse en cuestión elementos implícitos que forman parte del hacer, decir, sentir y pensar docente (Monereo y Badia, 2004).

\subsection{LOS INCIDENTES CRITICOS Y LA IDENTIDAD DOCENTE COMO ALTERNATIVAS PARA LA FORMACION PERMANENTE}

El profesor universitario al realizar su clase enfrenta un escenario muchas veces incierto, en el cual pone en juego su experiencia para dar respuesta a desafíos y demandas que con cierta frecuencia son imprevistas. A pesar de la riqueza de estos escenarios y las experiencias que en ellos ocurren, no necesariamente se obtendrá a continuación una sistematización de procesos y resultados, ni un aprendizaje profesional explícito, formalizado y comunicable, pues para ello será necesario un trabajo intencionado y un análisis de la situación que resulta clave.

En la medida que los eventos imprevistos se convierten en hitos relevantes tienen un impacto cognitivo, social y/o emocional, y ponen al docente en una situación conflictiva, se transforman en sucesos significativos y desestabilizantes, conocidos como incidente crítico (Navarro, et al., 1998). En consecuencia, cuando ocurre un incidente que el profesor ha 
significado como tal, su estabilidad personal cede terreno a la inseguridad, la inhibición y quizás a la agresividad, afectándole y poniendo en crisis su identidad, dado que no cuenta con los recursos que le permitan afrontar de manera oportuna y adecuada aquel incidente crítico, cuestionándolo en lo que ha dicho, sentido, pensado y/o realizado.

A partir de lo anterior, puede comprenderse que los incidentes críticos serán explicados como un evento relevante, cuyo enfrentamiento se derive de una respuesta intuitiva, espontánea, que incluso podría naturalizarse como parte de un repertorio esperable por parte de un profesor. Sin embargo, de ser entendidos así, se les restarían a estos eventos las posibilidades de convertirse en un recurso aprovechable en la formación permanente del profesorado.

Particularmente, en el contexto de esta investigación los incidentes críticos son entendidos como sucesos que acontecen en un tiempo y espacio determinado y que al significarse como negativos superan cierto umbral emocional, ponen al docente en conflicto y le desestabilizan, de modo que para sobreponerse, recuperar y continuar con su tarea no le bastará una estrategia local, sino que necesitará utilizar mecanismos psicológicos profundos, que afecten su identidad y que por tanto configuren una nueva o nuevas versiones de sí mismo (Monereo, Badia, Bilbao, Cerrato y Weise, 2009).

En términos conceptuales la identidad se ha definido a partir de preguntas como: ¿Quién o quiénes soy como profesor?, ¿cuáles son los significados vinculados a mí mismo en mi trabajo?, ¿qué tengo que hacer y por qué? (Day, 2006). En el mismo sentido, las respuestas a estas preguntas abordarán dimensiones de la identidad que han sido descritas en la literatura, y que aluden a roles profesionales, las concepciones y principios sobre la enseñanza y el aprendizaje, las estrategias utilizadas y los sentimientos asociados a la labor docente (Monereo y Badia, en prensa).

Por otro lado, en relación a la formación del profesorado Martin y Cervi (en Pozo, 2006) señalan que la comprensión implícita de un incidente crítico como ha sido descrito, sólo permitirá un uso técnico del conocimiento derivado de ello, mientras que su explicitación facilitaría que el profesor pudiera desarrollar su experiencia para hacer un uso estratégico de ese conocimiento, y de esta manera ajustar sus decisiones y su práctica al complejo mundo de la sala de clases.

A partir de lo anterior, se comprende que el profesor debe tomar decisiones dentro de su actividad como enseñante, en las cuales situaciones experimentadas previamente toman un papel decisivo, en la medida que estas experiencias sean incorporadas en la toma de decisiones y el aprendizaje profesional. En la práctica, si se produce un incidente crítico, el profesor podrá poner en marcha un proceso de autorregulación (sin necesariamente interrumpir su tarea) generando así nuevo conocimiento y el despliegue de numerosos recursos de respuesta cognitiva, emocional y comunicacional ante el incidente ocurrido. Sin embargo, desarrollar este potencial implica crear oportunidades para reflexionar, promover el cambio y aprender nuevas estrategias de enfrentamiento, frente a lo cual un análisis sistemático y pormenorizado de los incidentes críticos que ocurren en el aula permitirá a los docentes anticipar sus respuestas y ampliar el repertorio de acciones a las que acuden cuando atraviesan una situación compleja en la interacción con sus estudiantes y/o con los contenidos de enseñanza.

Desarrollar procesos reflexivos para la planificación, regulación y evaluación de la enseñanza puede producir mejoras en ciertas prácticas pedagógicas, sin embargo no por ello supondrá un cambio en las concepciones y sentimientos profundamente arraigados en el profesional, más si consideramos que aquello es parte de su identidad como enseñante 
(Monereo y Badia, en prensa). En consecuencia, el cambio en la formación permanente del profesorado universitario deberá ser entendido a partir del desarrollo y transformación de su identidad profesional como docente (Settlage, 2009).

Frente a esta realidad, resulta complejo y a la vez necesario explorar en las posibilidades y contextos de desarrollo de los profesionales académicos que comienzan a responsabilizarse por la formación universitaria. Particularmente en el marco del presente estudio, el foco se centra en quienes son los responsables de enseñar en la formación inicial docente, bajo la perspectiva de aportar información relevante para la creación de propuestas en la formación permanente, que posibilite a los docentes universitarios analizar y valorar sus conocimientos, creencias y prácticas para el mejoramiento de la enseñanza, el aprendizaje propio y el de sus estudiantes.

\section{METODOLOGIA}

\subsection{DISEÑOY OBJETIVOS}

La presente investigación corresponde a un estudio cualitativo de carácter descriptivointerpretativo, en coherencia con el análisis de los discursos de los sujetos participantes. A través de entrevistas se profundizó y analizó la información como un medio para alcanzar los propósitos planteados. A continuación se expone el objetivo general definido para esta investigación:

- Analizar los componentes emergentes en la identidad docente de los profesores universitarios que realizan clases en la formación inicial docente, así como los incidentes críticos que han enfrentado en su experiencia como formadores en pregrado.

En consecuencia con el objetivo general, la investigación se focaliza en el logro de los siguientes objetivos específicos:

1) Identificar los componentes de la identidad docente que surgen a partir del relato de los profesores universitarios en relación a sus concepciones sobre, metodologías, roles y sentimientos asociados a su experiencia y formación como académicos.

2) Describir las temáticas relacionadas con la emergencia de los incidentes críticos.

3) Caracterizar ejemplos de incidentes críticos surgidos en clases de formación inicial docente y las formas más típicas en que los docentes universitarios enfrentan dichos incidentes.

Los objetivos aquí planteados responden a una investigación inicial, en la cual se ha considerado una muestra intencional que permita la consecución de los propósitos y resultados a los que se aspira.

\subsection{MUESTRA}

La muestra de este estudio corresponde a cinco profesores que trabajan en distintas universidades chilenas. Como criterios homogeneizadores de la muestra, se definió que 
fueran docentes que imparten clases en la formación inicial docente, con un máximo de seis años de experiencia en esta labor, definiéndoles de esta manera como profesores noveles. Junto con esto se pudo establecer una edad promedio de 41 años.

Respecto a la formación previa, tres de los docentes se formaron como pedagogos, uno como licenciado en Historia y un quinto como Psicólogo. La mayoría de los profesores están en proceso de formación de postgrado en alguna disciplina del ámbito educativo, en tanto que uno tiene el grado de doctor en educación.

Tabla 1

Datos de la muestra

\begin{tabular}{|l|l|l|l|}
\hline \multicolumn{1}{|c|}{ Género } & \multicolumn{1}{|c|}{$\begin{array}{c}\text { Grado } \\
\text { académico }\end{array}$} & $\begin{array}{c}\text { Experiencia de docencia } \\
\text { en pedagogía }\end{array}$ & \multicolumn{1}{c|}{$\begin{array}{c}\text { Nivel en que imparte } \\
\text { docencia }\end{array}$} \\
\hline Femenino & Doctor & Cuatro años & Grado \\
\hline Masculino & Candidato a doctor & Seis años & Grado y postgrado (en otra área) \\
\hline Femenino & Candidata a doctor & Seis años & Grado \\
\hline Femenino & Magister & Cinco años & Grado \\
\hline Femenino & Candidata a doctor & Seis años & Grado y Postgrado \\
\hline
\end{tabular}

\subsection{PROCEDIMIENTOS DE REGISTRO Y UNIDADES DE ANALISIS}

El procedimiento seguido luego de la elaboración del protocolo de preguntas en relación con el rol profesional, enfoques educativos, estrategias docentes y sentimientos sobre la docencia, fue realizar entrevistas individuales semiestructuradas, cuatro de las cuales se realizaron por vía telefónica y una de ellas de manera personal. Todas las entrevistas fueron grabadas en audio y transcritas a textos digitales, para su análisis posterior.

En coherencia con el carácter cualitativo de esta investigación, la organización y análisis de los datos se adhieren a los postulados de la Grounded theory o Teoría emergente (Briones, 2005). En consecuencia, se realizó el análisis de contenido a partir del establecimiento de las unidades y reglas de análisis para luego definir categorías, procediendo posteriormente a la discusión e interpretación de los datos recogidos (Cáceres, 2003). Como unidad de análisis se definió a las secuencias discursivas de carácter descriptivo, argumentativo o explicativo que se referían a algún aspecto de la identidad docente, considerándose como componentes temáticos o siguiente: roles, principios y concepciones educativas, estrategias y sentimientos asociados a la enseñanza, así como también aquellos aspectos referidos a incidentes críticos y a la formación permanente del profesorado universitario.

En el caso de este estudio es deber señalar que las categorías elaboradas se definen como conjuntos temáticos de carácter general, en los cuales se integran las unidades de análisis seleccionadas. Estas categorías tienen sustento en dos fuentes relacionadas, pero distintas en su naturaleza, dado que unas categorías emergen desde la discusión bibliográfica (previas), en tanto otras fueron elaboradas a partir del análisis de los datos (emergentes). 
Finalmente, para el análisis de contenido se ha utilizado el programa Atlas.ti (Muhr, s.f), principalmente porque resulta una herramienta útil, que facilita la organización y revisión de datos cualitativos, así como una interpretación rigurosa y dinámica.

Luego de una primera elaboración de categorías se realizó un proceso de fiabilidad interjueces. Este proceso consistió en una revisión de los segmentos seleccionados (unidades de análisis) y su representación en los conjuntos y categorías donde fueron integrados. Posteriormente a esta revisión realizada por dos jueces pares, se procedió a una reorganización y descarte de algunas categorías en aquellos puntos observados como débiles, ya fuera en términos de su conceptualización y/o contenidos temáticos (subcategorías).

\section{RESULTADOS}

\subsection{LOS COMPONENTES DE LA IDENTIDAD}

A continuación se presenta el primer nivel de análisis realizado, desde el cual se obtiene una red categorial con conjuntos temáticos específicos. Cabe señalar que el proceso de construcción fue de carácter emergente e inductivo, y los resultados fueron producidos a partir de las unidades de análisis elaboradas. En coherencia con el primer objetivo específico del estudio se procedió a identificar los componentes en la identidad docente, que se exponen en las categorías elaboradas mediante un cuadro que las resume, siendo todas las de carácter excluyente (Tabla 2).

Tabla 2

Resumen de categorías producidas

\begin{tabular}{|c|c|}
\hline Categorías sobre las representaciones & Conjuntos temáticos \\
\hline $\begin{array}{l}\text { 1. Enseñar y aprender en la } \\
\text { universidad }\end{array}$ & $\begin{array}{l}\text { 1.1. En búsqueda de un referente } \\
\text { 1.2. Un docente y sus múltiples roles } \\
\text { 1.3. Vínculos y distancias entre los distintos roles } \\
\text { 1.4. El docente universitario como modelo }\end{array}$ \\
\hline $\begin{array}{l}\text { 2. Metodologías y estrategias en las } \\
\text { clases de FID }\end{array}$ & $\begin{array}{l}\text { 2.1. Enseñando con utilidad y sentido } \\
\text { 2.2. Formando a futuros maestros } \\
\text { 2.3. La planificación y los elementos emergentes }\end{array}$ \\
\hline $\begin{array}{l}\text { 3. Sentimientos asociados a la } \\
\text { docencia }\end{array}$ & $\begin{array}{l}\text { 3.1. El gusto por la docencia universitaria } \\
\text { 3.2. La experiencia, la incertidumbre y sus sensaciones }\end{array}$ \\
\hline $\begin{array}{l}\text { 4. La formación permanente } \\
\text { del profesorado universitario }\end{array}$ & $\begin{array}{l}\text { 1.1. La autoformación desde un contexto informal } \\
\text { 4.2. Las oportunidades desde lo institucional }\end{array}$ \\
\hline
\end{tabular}

A continuación, con el propósito de orientar en la comprensión del procesamiento de los datos se entrega para cada una de las categorías elaboradas una definición y un 
segmento ejemplar seleccionado de las entrevistas. Cabe agregar que esta elaboración resultó muy útil en los procesos de fiabilización y revisión de las categorías.

A) Enseñar y aprender en la universidad: Esta categoría toma elementos distintivos en relación con representaciones sobre la enseñanza universitaria, y sobre los roles que desempeñan o quisieran desempeñar los profesores universitarios.

-1A. En búsqueda de un referente: Representaciones expresadas en el discurso y que en ocasiones tienen relación con el enfoque constructivista de los procesos enseñanza y aprendizaje. Las alusiones realizadas por los profesores se clasifican como genéricas y no necesariamente tienen un referente práctico o metodológico en sus clases.

“...una de las cosas que tengo que hacer es ver qué está pasando y cómo están pensando, yo creo que eso parte, y ahí hay un referente muy vigotskyano en mí en términos de aproximarme de trabajar en temas de zonas de desarrollo próximo..." (Docente 5).

-2A. Un docente y sus múltiples roles: Representaciones sobre las distintas funciones o roles asumidos por el profesor universitario en la actualidad y que suelen generar tensión debido a la complejidad y el tiempo requerido para responder de manera adecuada en cada una de las tareas que estas funciones implican.

"Mira, ojalá yo te pudiera decir que son complementarias, que sería bueno. De repente yo siento que me absorbe mucho tiempo la gestión, que me quita tiempo como para seguir estudiando, para preparar mejor mis clases, para dedicar a la investigación. No te podría decir que es un complemento ideal; de repente me veo sobrepasada por el tiempo" (Docente 4).

-3A. Vínculos y distancias entre los roles del profesor universitario: Representaciones acerca de la valoración, en términos positivos y negativos, de las diferentes funciones asociadas al desempeño de los profesores universitarios y la relación entre éstas.

"Si, todos los resultados o todos los aprendizajes que van surgiendo en el ámbito de la investigación si los llevo siempre a la clase, y también el tema de las tutorías en el sentido de identificar cuáles son las dificultades que los alumnos están teniendo porque eso también te ayuda" (Docente 1).

4A. El docente universitario como modelo: Representaciones acerca del profesor universitario de pedagogía como un ejemplo en la clase. Esto implica que el profesor se asume en un rol demostrador de buenas conductas y actitudes, las cuales a través de este modelado pueden ser desarrolladas por sus estudiantes. Las conductas o actitudes pueden estar referidas a formas de reflexión, de pensamiento, de relación con otros, hábitos laborales, entre otros.

“...un ingeniero, por ejemplo, no tiene por qué demostrarles a los otros estudiantes qué es ser un buen profesor; en cambio, uno tiene que ser un buen profesor, y en ese sentido creo que hay un matiz didáctico, importante..." (Docente 2).

B) Metodologías en las clases de formación inicial docente: Esta categoría aborda aquellos aspectos que se relacionan con los procedimientos y estrategias que usan o consideran relevantes los profesores en sus clases con los futuros maestros. 
-1B. Enseñando con utilidad y sentido: Representaciones acerca de una enseñanza con contenidos relevantes y vinculados al contexto de práctica o al desempeño laboral del futuro profesor. En el discurso implica que los contenidos a enseñar deben dar respuestas a las tareas concretas que el maestro en formación deberá enfrentar, definiéndose como fundamental dentro de las tareas docentes.

“...entonces siempre trato de articular teoría con práctica, y como ellos también van viviendo el proceso directamente relacionado a experiencias laborales, inmediatamente voy haciendo los procesos, cosa que vivan la experiencia y además la miren desde el contexto teórico y además la miren desde los contextos prácticos, y vayan aplicándolo ojalá inmediatamente u observando también la realidad..." (Docente 5).

-2B. Formando a futuros maestros: Representaciones que refieren a elementos específicos $\mathrm{y}$ distintivos en la formación de futuros profesores. Estos elementos consideran cuestiones relevantes en la formación y que desde el discurso se proponen integrar en sus clases.

“.... siento que eventualmente los cabros tienen que ser buenos lectores, tienen que ser críticos, tiene que ser, tienen que aprender a reflexionar, tratar de no sólo de digerir, sino que problematizar, qué sé yo..." (Docente 4).

-3B. La planificación y los elementos emergentes: Representaciones acerca de lo que los profesores se proponen realizar en la clase para conseguir los aprendizajes, ya sea como actividades o metodologías de enseñanza. Esta planificación se menciona desde una perspectiva general, como parte de una práctica común, que considera los elementos que emergen durante la clase.

"Yo ocupo la modalidad de debate, debate virtual, mientras estamos en clases, estamos en paralelo discutiendo en un foro virtual, entonces en ese foro virtual aparecen muchos temas, entonces los chicos empiezan a darle muchas vueltas a un tema en la discusión, entonces ese tema lo traemos a la clase y ahí modificamos la estructura, yo no tengo una estructura rígida, tengo una planificación inicial, pero yo diría que de todos los semestre nunca la he hecho tal cual como la planifiqué" (Docente 2).

C) Sentimientos asociados a la docencia: Esta categoría aborda aspectos afectivos o emotivos en relación a la docencia, ya sea en términos significados por el profesor como negativos o positivos.

-1C. El gusto por la docencia universitaria: Manifestaciones en el discurso que expresan las preferencias por las tareas y el rol de la docencia en el ámbito universitario. Estas preferencias están marcadas por representaciones positivas frente a los desafíos de enseñar y trabajar con los estudiantes.

"Sí, mira, tengo en este minuto sólo seis horas de docencia directa que yo quise mantener, porque a mí me encanta estar en el aula y el resto de mi jornada está dedicada a la dirección de innovación, estoy terminando el doctorado...” (Docente 3).

-2C. La experiencia, la incertidumbre y sus sensaciones: Manifestaciones asociadas en el discurso a la experiencia docente actual y pasada, en relación a cómo se experimenta la incertidumbre y la certeza en ciertas situaciones educativas o en las relaciones interpersonales dentro del aula. 
“...de pronto había algunas preguntas de cosas que de verdad no sabía, no me acordaba bien, entonces a la clase siguiente buscaba la información y les daba la información de lo que me habían preguntado, pero era una sensación incómoda porque sentía que no tenía el tiempo suficiente para preparar la clase" (Docente 1).

E) La formación permanente del profesor universitario: Esta categoría aborda representaciones en relación a como los profesores interpretan y definen las condiciones de la formación permanente en el contexto universitario, abordando las limitaciones, ventajas y consecuencias que actualmente vislumbran.

-1E. La autoformación y la formación desde un contexto informal: Concepciones acerca de la formación del profesorado universitario como un camino en solitario y en donde se vislumbran pocos o escasos espacios colegiados y formales de aprendizaje docente.

\begin{abstract}
"Bueno, de hecho yo creo que tendríamos que abordar todo este tipo de situaciones y pensar qué otro tipo de situaciones podrían ocurrir, y pensar en las estrategias para enfrentarlos adecuadamente, porque en el fondo tú cuando las vas viviendo, te vas dando cuenta que pasan y a veces cuando vas escuchando a otros colegas que les pasan otras cosas, tú también vas aprendiendo de otras experiencias, pero aprendes de ellas como anécdotas, pero no cómo enfrentarlas..." (Docente 1).
\end{abstract}

-2E. Las oportunidades desde lo institucional: Representaciones acerca de algunos espacios formales, que ofrecen un potencial no necesariamente utilizado para la formación del profesorado universitario en el rol docente.

"Hay consejos de instituto, por lo menos una vez al mes, y en esos consejos en este caso por escuela docente, la idea es tratar temáticas que nos estén..., ahora últimamente ha sido poco eso porque estamos enfrascados en el proceso de acreditación, entonces todo es acreditación" (Docente 3)

\title{
3.2. LAS TEMATICAS Y ESCENARIOS QUE INFLUYEN EN LOS INCIDENTES CRITICOS
}

Dentro de los objetivos de este estudio se planteó un trabajo en relación a describir los escenarios y formas de enfrentamiento de los incidentes críticos, trabajo al que se le añadió una dificultad particular en la medida que estos eventos tendían a ser vistos como anécdotas que no se sistematizan, por lo que se dificultaba su recuperación en detalle. A pesar de esto, en el análisis de los incidentes que se explicitan, se pudieron identificar tres temas interesantes en relación a su interpretación:

a) Cuando un incidente crítico cuestiona al docente: Surge de las representaciones del docente acerca de lo que se quiere o puede modificar luego de ocurrido un incidente. Esto implica la reflexión inmediata o posterior en relación a ciertas situaciones desestabilizantes ocurridas durante la clase.

“...igual he usado otras, lo que pasa es que con el tema de los textos usé siempre la misma, pero ahora con los textos tendré que buscar otra metodología porque el tema de que lo traigan leído no funciona, igual tienen que leer, les paso cosas cortas, ocupar trabajo en parejas donde vayan leyendo de a una parte y la vayan 
conversando, extrayendo las ideas principales, tengo que buscar más estrategias de lectura" (Docente 1).

b) Cuando el incidente crítico es visto como una consecuencia: Surge desde una visión o representación acerca de los incidentes como secuela directa de las metodologías innovadoras o recientemente introducidas por los profesores en la clase.

“...siempre los incidentes con los alumnos tienen que ver con quejas respecto de algo que a ellos no pueden o no alcanzan a hacer, o no les gusta hacer el tema de la lectura, el tema de escribir, no les gusta escribir, y justamente lo que yo hago es todo lo contrario, aquello que no les gusta, no todos, pero efectivamente porque lo requiero, porque si no, no sé cómo están organizando su pensamiento..." (Docente 5).

c) Respecto de las situaciones donde ocurren los incidentes críticos: Las representaciones acerca de cuándo ocurren los incidentes en el aula abordan distintos temas, sin embargo pueden encontrarse elementos comunes en términos de situaciones, implicaciones e hipótesis explicativas de los profesores, las cuales serán abordadas más adelante. Un ejemplo de ello se rescata en el siguiente segmento.

“...yo creo que mi mayor dificultad tiene que ver con la evaluación, las mayores crisis que he tenido con los alumnos, porque en términos de las relaciones y otras cosas a nivel general nunca he tenido dificultades, y a lo mejor debiese yo ser más clara, que ellos tengan claridad de cómo voy a evaluar, o a qué cosas apuntar, como para evitar un poco esa situación” (Docente 1).

\subsection{LOS INCIDENTES CRITICOS, CARACTERISTICAS Y ENFRENTAMIENTO}

Como tercer objetivo de este estudio se propuso una caracterización de los incidentes críticos que pudieran servir de representación sobre lo que ocurre en el aula. A partir de su recopilación dentro de este estudio, se ha podido elaborar la siguiente clasificación:

- Los incidentes y su origen: Frente a la posibilidad de identificar las situaciones o variables comunes que influyen en los incidentes, los docentes sugieren al menos cuatro factores que desde su comprensión resultarían relevantes: El perfil del estudiante que responde más bien a un sujeto resistente y/o receptivo, los momentos de evaluación como espacios especialmente sensibles en que emergen conflictos o problemas, la escolarización previa que no prepararía al estudiante para la vida universitaria y como ya se ha mencionado, la introducción de metodologías o innovaciones, frente a las cuales los alumnos no se sienten a gusto o no están habituados. A continuación dos citas que ejemplifican la asociación antes señalada:

“...en la evaluación que me hicieron (los estudiantes) al final de semestre se quejaron de que era mucho taller, querían más clases expositivas, pero eso, eso en la carrera lo hemos conversado, que les pasa en realidad también a otros profesores que los alumnos no quieren hacer nada en la clase, quieren que tú les des todo hecho y esa es una dificultad también que como carrera tenemos que enfrentar porque la pasividad de repente de los alumnos es mucho..." (Docente 1). 
"Con la evaluación, ahí está el meollo del asunto, prácticamente es lo que más les preocupa a los estudiantes, porque además lo que pasa es que no, no es que yo sea el innovador o el profe excelente, pero yo les cambio un poco el switch, por ejemplo no les hago prueba, no les pido informes, no les corrijo el portafolio, lo autoevaluamos; hacemos dos aperturas durante el semestre, ¿te fijas..., entonces les cambia un poco del modo tradicional del que vienen ellos" (Docente 2).

- Los incidentes como una posibilidad inevitable: Los incidentes críticos son vistos como parte del escenario natural en el cual se trabaja, entendiendo que donde hay interacciones y un trabajo conjunto que depende de varios sujetos su ocurrencia resulta más que probable. En relación a esto se ofrecen dos citas como ejemplo:

“...Yo creo que uno siempre los vive, todos los años, y dependiendo también de los grupos que te tocan, lo cierto es que cuando inicié el trabajo con los docentes los desequilibrios eran enormes, una porque yo creo que no me centraba tanto en los procesos de aprendizaje de ellos..." (Docente 5).

"Siempre es el tema del tiempo, es decir, cuando estoy haciendo la clase me doy cuenta que con las actividades que estaban planificadas no me va a alcanzar el tiempo, y que tiene que ver también con que los estudiantes de repente hacen las cosas más lento, o que yo me extiendo en el discurso, o que los estudiantes preguntan más...” (Docente 2).

- Los incidentes y su naturaleza: El incidente crítico como evento suele asemejarse con un conflicto de naturaleza interpersonal, con tintes de agresividad y relacionado de manera focalizada con el clima del aula o la relación entre profesor y estudiante, reduciendo de esta manera su significado e implicaciones. A seguir, un par de ejemplos:

"no, yo en ese contexto, no me han tocado problemas disciplinarios; no, no tengo problemas en ese sentido, y creo que es porque cuando yo parto, el primer mes, con los estudiantes negocio bastante la forma de trabajo... ya están a punto de egresar, entonces problemas de comportamiento no, alguna rudeza, tampoco, yo también trato de ser muy respetuosa con mis estudiantes..." (Docente 3).

"Mira, conflictivo así como confrontacional nunca me ha pasado, conflictivo confrontacional, nunca,...” (Docente 4).

- Los incidentes como una oportunidad potencial: Si bien es cierto no se puede hablar de un trabajo sistemático o un análisis pormenorizado de los eventos significados como incidentes, puede encontrarse una mirada reflexiva respecto del potencial que ofrece un incidente para el aprendizaje profesional docente, como lo señala el siguiente ejemplo:

“...te digo me da risa porque ya lo tomo como algo que sirve, como un hecho de que nos es útil para algo. Me alegra de repente que me ocurran estas cosas, ya no es como antes que me complicaba y decía ¡uy!, ¿que va a pasar? Estos tipos se van a ir donde el vicerrector. Me generaba mucha inseguridad, me generaba conflictos personales, pero la experiencia me ha hecho reflexionar al respecto y ya lo considero como un evento de aprendizaje" (Docente 2).

"No, me encantaría que me hubiera pasado, pero no, tienden a ser muy pasivos los chicos..." (Docente 3). 
En resumen, a partir de los resultados en relación a los objetivos específicos 2 y 3 propuestos, se puede contar ya con una evidencia inicial, que permite rescatar variables para un trabajo de profundización a nivel de incidentes críticos ocurridos en el aula. Sin embargo, se cree importante poder reflejar a los profesores la complejidad y multidimensionalidad de estos eventos. Con el propósito de ilustrar los aspectos mencionados en este punto se ha elaborado un inventario con algunos ejemplos inspirados en los incidentes críticos enfrentados por los docentes participantes del estudio (Anexo).

\section{DISCUSION E IMPLICACIONES FUTURAS}

A modo se sintetizar los resultados explicitados, y en respuesta a los objetivos planteados, a continuación se discuten los principales hallazgos e implicaciones derivadas de este estudio:

En relación a la conformación de la identidad docente, entendiéndola desde las preguntas sobre, ¿quién o quiénes soy como profesor?, ¿cuáles son los significados vinculados a mí mismo y mi trabajo?, ¿qué tengo que hacer y por qué? (Day, 2006), se define que: Los profesores universitarios que componen la muestra se ven a sí mismos como modelos dentro de la clase. Esto implica que son un reflejo de lo que quieren que sus estudiantes piensen, hagan y sean. En relación a elementos distintivos de su propia especialidad, los docentes refieren diferencias entre hacer clases en pedagogía, respecto de otras carreras. Particularmente establecen que enseñar a futuros profesores implica desarrollar una buena enseñanza, articulando la teoría y la práctica, haciendo un modelaje que ejemplifica lo que es lo esperable de un buen profesor, todos elementos considerados relevantes en su construcción como docente universitario. En este sentido, el papel de ellos como modelos en el conocimiento, procedimientos y actitudes implica ciertas convenciones respecto a qué significa ser un buen profesor de futuros maestros. Además este discurso se relaciona con un compromiso social por la tarea docente y la aspiración de impactar, a través de su enseñanza, en el sistema educativo, mediante la formación de buenos maestros, denotando con esto un componente ideal en su identidad, frente a un rol social más amplio.

Por otro lado, en relación a su trabajo en la institución, los docentes entrevistados desarrollan en algún grado funciones de investigación, gestión y docencia, la multiplicidad de estas funciones o roles es algo que les preocupa. Frente a esto la función que aparece como menos atractiva es la de gestionador, lo cual implica que esta función es vista más bien como secundaria y desconectada de las otras funciones. Mayor es el valor del vínculo entre investigación y docencia, en donde los profesores esperan una retroalimentación que les permita mejorar, plantearse preguntas y nutrirse en ambas funciones.

En coherencia con lo anterior, dentro de las funciones como profesor la mayoría se siente más cómodo haciendo clases en pregrado (formación inicial), pudiendo esto ser atribuido a distintos significados como, estar en contacto con nuevas generaciones, el compromiso social y la relevancia de su función frente a las posibilidades de cambio en educación. Sin embargo, en relación a las expectativas frente a su alumnado, destaca la posibilidad que otorga hacer clases en grupos de estudiantes de último año o en postgrado, en donde se facilita una profundización y un cambio cualitativo en los temas a trabajar. 
Por otro lado, a diferencia de lo que la literatura especializada señala (De la Cruz, Pozo, Huarte et al. 2006), el docente como profesional experto es algo ausente y sólo se menciona a partir del recuerdo de algunas experiencias formativas previas a la docencia universitaria. En términos de esto, a pesar que no todos los docentes provienen de una formación de origen como pedagogos, se asumen como enseñantes, y es ésta la profesionalidad que emerge permanentemente en el discurso, frente a esto cabe preguntarse si el trabajar en facultades de pedagogía les impregna de una identidad colegiada.

Un aspecto que surge como crítico es la confusión o ausencia de referentes teóricos claros en relación a la enseñanza. Sólo el constructivismo aparece en ciertas ocasiones como una perspectiva teórica adoptada en el discurso de los docentes, ya sea como algo que se proponen actualmente o como modelo de instrucción al cual aspiran. Sin embargo su aparición es bastante reducida y aislada. En general no se explicitan con suficiente claridad o especificidad las perspectivas teóricas o principios educativos que guían el actuar y decisiones, por lo cual es difícil identificar estrategias o metodologías que tenga un referente teórico explícito. Dentro de este mismo ámbito se establece que una parte importante de la enseñanza debe planificarse, rescatando desde aquí dos elementos que emergen como erráticos, el uso abusivo de algunas metodologías y que los docentes asuman responsabilidades que en principio son planteadas para los alumnos, considerando esto como parte de una respuesta frente al imprevisto, asociando ambas situaciones a incidentes críticos.

En relación a los componentes afectivos y los sentimientos, se puede reconocer que el compromiso, el aporte social de formar futuros maestros y las posibilidades de mejora visualizadas son elementos que emergen permanentemente. En términos negativos el análisis de los datos evidencia cierta insatisfacción sobre elementos que resultan difíciles de cambiar como algunas condiciones institucionales, la formación previa del estudiantado (percibida como débil), y la percepción de la propia competencia en el enfrentamiento de los incidentes críticos. La relación entre estos dos últimos puntos se refleja en la imagen que se tiene del estudiante y los conflictos que debe enfrentar el profesor al trabajar con ellos en el aula, particularmente cuando existe una visión predominante de que estos estudiantes no reúnen los requisitos para desarrollar el proceso de enseñanza-aprendizaje, cuestión que genera frustración e inquietud en el profesorado.

En relación al proceso de formación seguido a partir de la elección de la carrera docente, ellos se definen a sí mismos como sujetos que se han construido más bien en solitario, y que a partir de la docencia universitaria han seguido una formación informal y alejada de lo colegiado. De no ser por los postgrados, no se podrían distinguir experiencias de formativas claras y focalizadas en la docencia universitaria. En este mismo sentido, además de asumir distintas funciones deben buscar oportunidades para la propia formación.

Al buscar hipótesis sobre el origen de los incidentes críticos, entendiéndolos como eventos que en este caso emergen en el aula, y que desde el punto de vista del docente lo desestabilizan emocional y/o cognitivamente (Monereo, Badia, Bilbao, Cerrato y Weise, 2009), se construyen al menos tres explicaciones que se focalizan en los alumnos: Primero, una imagen de un estudiante que tiende a la pasividad y/o la resistencia, cuestión que a juicio de los profesores debilita la participación, el compromiso, y les aleja de una postura reflexiva y crítica, elementos considerados importantes por los docentes y su forma de enseñar. Segundo, las barreras para el aprendizaje enfrentadas por 
los estudiantes, lo cual se relacionaría con debilidades en los aprendizajes previos y una historia escolar que no les prepara para la universidad, desde esta imagen del alumnado surge como hipótesis explicativa la idea de que los alumnos aprendieron a trabajar bajo ciertas lógicas que en el presente no se acomodan a lo que los profesores universitarios les exigen. Tercero, algunos planeamientos sugieren que las metodologías de evaluación utilizadas y la introducción de innovaciones en las formas de trabajo crearían distancias entre las expectativas de los alumnos y las de los docentes, provocando crisis. Siendo así, se puede establecer que dadas las explicaciones anteriores los profesores esperarían que sus alumnos tuvieran una actitud más madura, comprometida y con una participación más activa, pero para lo cual no estarían suficientemente preparados.

En base a lo anterior puede señalarse que, desde la perspectiva de los profesores, sus estudiantes se sentirían más cómodos en clases expositivas, actuando como receptores de contenidos, es decir, desde una concepción de enseñanza entendida como instrucción directa. De la misma manera esto influiría en su resistencia a ciertos cambios o innovaciones metodológicas asociados a los procedimientos e instrumentos de evaluación, actividades grupales y la introducción de innovación en la clase. De esta forma se crea una asociación entre déficit en aprendizajes previos receptividad y resistencia; sin embargo, al respecto cabría profundizar en la relación entre lo que perciben los profesores sobre sus estudiantes y la imagen que tienen de la educación previa que han recibido.

Por otro lado, el conflicto en el incidente crítico responde más bien a una situación no sistematizada, experimentado a veces como extremo, y con una lectura más bien externa e individual de las variables que lo influyen. En el mismo sentido, su enfrentamiento aparece de manera más bien intuitiva, asumiendo que hacerse cargo de ello es parte de las tareas docentes y desde donde surgen respuestas o intervenciones con un enfoque individual o reparador de la urgencia. Desde esta postura, resulta complejo asumir una mirada sistémica de los incidentes, para un enfrentamiento de corte más integrador, estratégico y formador, tanto en el caso de los profesores, como en el de sus estudiantes.

En relación a los componentes emotivos de la identidad, se puede señalar que probablemente tener que recordar una imagen negativa de sí mismo, reconocer que se cometieron errores o no se supo actuar adecuadamente, puede influir en una cierta omisión de los episodios considerados como críticos. En esto es deber reconocer que como cualquier profesional frente a este tipo de situaciones los profesores lo pasan mal, y en ocasiones al relatar un incidente terminan narrando casos de sus alumnos, los cuales han sido explicados a partir de dificultades que requieren más apoyo individual.

Desde el punto de vista de la formación permanente, se puede confirmar que existe una necesidad a ser atendida, particularmente en el contexto de una formación que responda a lo que los docentes enfrentan en el día a día. Si bien es cierto los profesores que participan de este estudio reconocen el potencial de la reflexión como instrumento de formación, puede evidenciarse desde los relatos que este proceso suele implicar un cuestionamiento, pero eso no conlleva un cambio en las representaciones, ni una guía para las formas de actuación en el aula (Martin y Cervi, 2006). Sin duda los procesos reflexivos son una parte importante de cualquier programa de formación, pero éste además deberá hacer frente a un desafío mayor, que es el cambio en las representaciones, sentimientos y prácticas profundamente arraigadas en el docente, de manera de impactar en su actuar en la forma en que toma las decisiones relativas a su docencia (Monereo y Badia, en prensa). 
Tal como señala Zabalza (s.f.), reconocido autor en el ámbito de la formación docente, en la enseñanza de la medicina se ha demostrado que los médicos noveles tienden a repetir los errores de sus maestros, cuestión que resulta sumamente problemática, no sólo en medicina, sino que en cualquier ámbito de la formación universitaria. Por consiguiente, si bien es cierto que resulta imposible impedir que los profesores cometan errores, pues es parte de la condición humana, sí se cree posible que la formación permanente se focalice en aprender de estos errores, evolucionar y a prevenir que ellos sean enseñados.

Respecto de los alcances y limitaciones, al finalizar es deber señalar que este estudio se ha propuesto con fines exploratorios y, por tanto, deberá ser entendido desde esta perspectiva. A partir de esto se cree necesario explicitar algunos aspectos particularmente relevantes y que han sido parte del proceso o se han evidenciado al finalizar el trabajo.

Frente a la complejidad de recoger información de carácter cualitativo, la entrevista resulta ser un medio bastante útil. Sin embargo se cree que un futuro estudio en esta línea se verá enriquecido a partir del uso de técnicas complementarias. Sin duda ampliar la recogida de información hacia la observación y registro de clases, grupos focales a docentes y entrevistas a estudiantes, entre otros, redundaría en una mejora sustantiva del proceso y resultados de investigación, ya que permitiría profundizar la mirada sobre el objeto-sujeto de estudio, contrastar la información y obtener mayor perspectivismo.

Otro de los asuntos que se debe enfrentar en este tipo de estudios es la complejidad de recoger eventos como los incidentes críticos. Más allá de las dificultades metodológicas, recoger estos episodios implica para los participantes asumir los propios errores, que se puede ser muy profesional y vulnerable a la vez y que eventualmente no se tienen las estrategias para enfrentar y evolucionar en el incidente, lo cual evidentemente involucra un componente emocional y/o cognitivo que debe tenerse presente.

En relación a la interpretación de los incidentes críticos, se cree importante poder reflejar a los profesores que un incidente puede ir más allá de un desencuentro de opiniones en clase, y que aunque refiere a un momento de conflicto puede abarcar una amplia gama de temas relacionados con su docencia y trascender la situación de aula, pues puede relacionarse con conflictos con los estudiantes, con sus colegas, con los contenidos y con la institución, entre otras variables complejas.

En relación a la formación permanente del profesorado, existe claridad que cualquier programa con estos propósitos debe plantearse desde un modelo teórico claro, en un contexto auténtico, con contenidos relevantes y considerando la diversidad de roles a los que debe responder el docente. En este sentido el uso de la anécdota y el potencial de las relaciones informales entre el profesorado puede transformarse en el punto de partida para una propuesta innovadora en la formación, sobre todo considerando el desafío que representa que los profesores se sientan identificados en sus necesidades de aprendizaje y puedan involucrarse desde un compromiso real.

Al finalizar queda agradecer la colaboración de los docentes universitarios que con su valioso tiempo y honestidad han participado de este estudio, y al equipo Identites-SINTE por su fundamental apoyo en la reflexión y revisión de este trabajo. 


\section{BIBLIOGRAFIA}

Avalos, B. (2002). Profesores para Chile, Historia de un proyecto. Santiago de Chile. Ministerio de Educación.

Badia, A., Monereo, C. (2004). La construcción del conocimiento profesional docente. Análisis de un curso de formación sobre la enseñanza estratégica. Anuario de Psicología, 1, (35): 47-70.

Badia, A., Monereo, C. (En prensa). Los heterónimos del docente: Identidad, selfs y enseñanza.

Benito, A., Cruz, A. (2005). Nuevas claves para la docencia universitaria en el Espacio Europeo de Educación superior. Narcea, S.A. Madrid.

Briones, J. (2005). El niño que se traslada y su relación con la lengua en la sociedad de acogida. Cap. 5, Análisis metodológico. Revista electrónica Redele. Recuperado el 12 de noviembre de 2009. http://www.educacion.es/redele/biblioteca205/briones/05.metodologia.pdf

Coll,C., Palacios, J., Marchesi, A. (2008). Desarrollo Psicológico y Educación. 2. Psicología de la Educación Escolar. Alianza: Madrid.

Cáceres, P. (2003). Análisis cualitativo de contenido: una alternativa metodológica alcanzable: Psicoperspectivas, 1 (1): 53-81.

Day, C. (2006) Pasión por enseñar. La identidad personal y profesional del docente y sus valores. Narcea, S.A: Madrid.

De la Cruz, M., Pozo, J., Huarte, M., Scheuer, N. (2006). Concepciones de enseñanza y prácticas discursivas en la formación de futuros profesores. En J. Pozo (coord.) (2006). Nuevas Formas de pensar la enseñanza y el aprendizaje. Las concepciones de los profesores y los alumnos. (pp. 359-371). Graó: Barcelona.

Martín, E., Cervi, J. (2006). Modelos de formación docente para el cambio de concepciones en los profesores. En J. Pozo (coord). Nuevas Formas de pensar la enseñanza y el aprendizaje. Las concepciones de los profesores y los alumnos. (pp. 419-434) Graó: Barcelona.

Monereo, C., Badia, A., Bilbao, G., Cerrato, M., Weise, C. (2009). Ser un docente estratégico: cuando cambiar la estrategia no basta. Cultura y educación, 21 (3), pp. 237-256.

Navarro, R., López, A., Barroso, P. (1998). El análisis de incidentes críticos en la formación inicial de maestros. Revista electrónica interuniversitaria de formación del profesorado. 1 (1).

Perales, M., Sánchez, P., Chiva, I. (2002). El "curso de iniciación a la docencia universitaria como experiencia de formación de profesores universitarios noveles en la Universidad de Valencia. Un sistema de evaluación. Revista electrónica de investigación y evaluación educativa, (8).

Perrenoud, P. (2007). Desarrollar la práctica reflexiva en el oficio de enseñar. Graó: Barcelona.

Settlage, J., Sutherland, S., Smith, L., Ceglie, R. (2009). Constructing a doubt-free teaching self: self -efficacy, Teacher Identity and science Instruction within diverse settings. Journal of research in science teaching, 1, (46): 102-125. 


\section{ANEXO \\ INVENTARIO DE INCIDENTES CRITICOS}

A continuación se presenta una serie de ejemplos inspirados en los relatos de los docentes participantes del estudio. Estos ejemplos de incidentes críticos obedecen a una selección realizada a partir de las entrevistas y se relacionan con distintos temas que pueden surgir en la sala de clases:

- Ejemplo 1: La jefa de carrera le ha solicitado a una docente hacerse cargo de un tema que no revisaba desde su época de estudiante. Al enfrentar las primeras clases, la profesora se siente muy insegura, a pesar de haber estudiado tiene el temor de cometer errores o que sus estudiantes le puedan preguntar sobre algo que no recuerde. En una de estas primeras clases y sintiéndose aún incómoda la profesora expone y una alumna realiza una pregunta sobre los planeamientos acerca de una teoría particular y conceptos específicos. La profesora responde improvisando y pensando en que seguramente se ha equivocado. A la siguiente clase la estudiante retoma el tema, y la profesora que ya se ha asegurado de su error no sabe qué decir.

- Ejemplo 2: Un profesor se ha propuesto hace tiempo incorporar el uso de foros virtuales en su asignatura. Para esto solicita a los estudiantes que revisen un tema y lo discutan durante tres semanas en paralelo a la realización de las clases. Muy a su pesar, cuando ya van desarrollando el segundo tema de la asignatura un estudiante se para en clase y manifiesta lo siguiente: "La semana pasada después de clase nos reunimos con un grupo de compañeros y decidimos pedirle que no haga más estos foros virtuales, nosotros preferíamos la discusión cara a cara, seguro aprenderemos más. Además no estamos de acuerdo con su forma de evaluación, nosotros preferimos estudiar, que nos tome exámenes y no andar escribiendo ensayos y haciendo portafolios".

- Ejemplo 3: La profesora ha decidido trabajar un texto usando la metodología puzzle y solicita a sus alumnos que se reúnan en grupos, con compañeros distintos a lo habitual, resaltando la importancia de aprender a trabajar con otros. Al acercarse y pedirle a una estudiante que se cambie de grupo, pues se ha sentado con los de siempre, se encuentra con la siguiente respuesta "Yo no entiendo por qué usted quiere desarmar los grupos, yo quiero trabajar con mis amigas". La profesora responde que hay que ser tolerante y valorar la diversidad lo cual la estudiante responde, "si usted me cambia de puesto lo haré, pero eso no significa que trabaje". La profesora confirma durante la clase que la alumna se mantiene en silencio y no trabaja.

- Ejemplo 4: La profesora ha distribuido el temario de la asignatura y preparado sus clases en base a unas lecturas que dio a sus estudiantes con suficiente anticipación. Cuando llega a la sala se encuentra con los estudiantes y les propone una guía para comenzar a trabajar, tras darse cuenta que varios alumnos hablan entre ellos, pregunta qué sucede y se da cuenta que la mayoría de sus estudiantes no han leído los textos. Frente a esto les propone hacer lecturas grupales y divididas, tarea que les lleva toda la clase, por lo que los estudiantes llevan las guías para trabajar en casa. A pesar de los compromisos adquiridos, la profesora se encuentra con que en la clase siguiente sucede lo mismo y se pregunta ¿qué hacer? 
